\title{
Building on Discovery: A Preview of 3DMS 2020
}

\section{Dorte Juul Jensen, Matthew P. Miller, and Lei Zhang}

The International Congress on 3D Materials Science (3DMS 2020) will meet for its milestone fifth iteration on June 28-July 1, 2020, in Washington, D.C. This renowned conference has helped to steadily advance 3D materials science and method development since it was first held in 2012. This summer, the meeting promises to build upon its eight years of discoveries and increase a burgeoning network of researchers and practitioners who are examining 3D microstructures of materials and are developing associated technologies and methods.

\section{Contributions of 3DMS to the Field}

The field has greatly advanced since the congress first met. By now, it is widely accepted that $3 \mathrm{D}$ characterization is generally needed as 2D methods may lead to insufficient and even misleading information. Several 3D methods and the associated data analysis tools have matured and are user-friendly. Various material properties can be predicted in terms of digitalized real 3D structure of materials by combining simulation and different constitutive relations. It is also shown that $3 \mathrm{D}$ data obtained by nondestructive methods, often using in situ capabilities at various sample conditions (for example at different times during annealing or plasticity), are becoming essential as input to and validation of various simulation tools.

These advancements will continue through discussions at 3DMS 2020. Topics will span 3D characterization, visualization, quantitative analysis, modeling, and development of structure-property relationships, along with the associated issues of big data and machine learning.

\section{Highlights of 3DMS 2020}

The planned technical sessions have been designed to showcase the broad work that researchers are doing to develop new techniques, technologies, and models. Plenary talks will feature three experts: Jaafar El-Awady, Johns Hopkins University, with "Acoustic Emission Measurements of Damage Accumulation and Crack Initiation in Metals at the Micron-scale;" Satoshi Hata, Kyushu University, with "Toward Dynamic 3D Visualization of Dislocations by Electron Tomography;" and Helena Van Swygenhoven, Paul Scherrer Institute and Swiss Federal Institute of Technology Lausanne, with "Operando and In Situ Synchrotron Experiments Following Microstructural Evolutions." The technical program also highlights invited speakers from China, Denmark, France, Germany, Japan, and the U.S.

Attendees will find tremendous value in making new acquaintances and reconnecting with other scientists, researchers, and engineers interested in 3D materials science and 3D method development. We expect more than 180 attendees at 3DMS 2020, so the potential to establish new collaborations is great. Every two years, the congress inspires materials researchers to create and implement 3D methods and thereby grow an international community.

What sets 3DMS 2020 apart from other similar meetings is its focus on characterization. Within all areas of the materials science field, the possibility to do characterization in $3 \mathrm{D}$ is a very important step forward. At 3DMS 2020, advanced 3D characterization tools with better spatial and temporal resolutions and new mathematical tools will be presented. An emphasis is placed on simulation of material behavior during processing or performance conditions. This
The 5th International Congress on

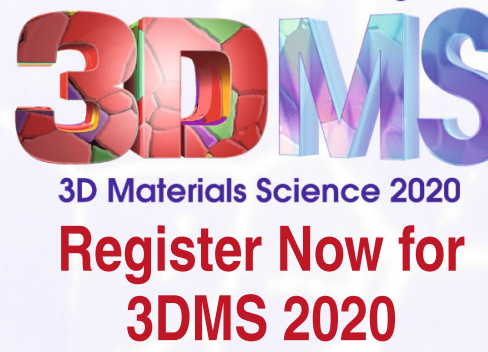

June 28-July 1, 2020 Hyatt Regency Washington on Capitol Hill, Washington, D.C., USA Increase your knowledge about the latest advances in 3D materials science while expanding your connections in this quickly evolving field. For cost-savings, register by the discount deadline of May 18. Note that the housing deadline to book the group rate at the Hyatt

is June 5. Don't miss itreserve your spot today. Visit www.tms.org/3DMS2020 for additional information and to register. 
area is undergoing a drastic evolution; powered by $3 \mathrm{D}$ characterization data, material models are being used for the first time to actually predict properties and performance, including the lifetimes of real industrial materials. An emerging capability is obtaining $3 \mathrm{D}$ characterization data dynamically during processes related to additive manufacturing. Machine learning and other data science methodologies will greatly enhance our ability to examine, digest, and understand these incredibly rich data sets in ways that can be most useful to process and, ultimately, to product design.

\section{The Value of 3DMS 2020}

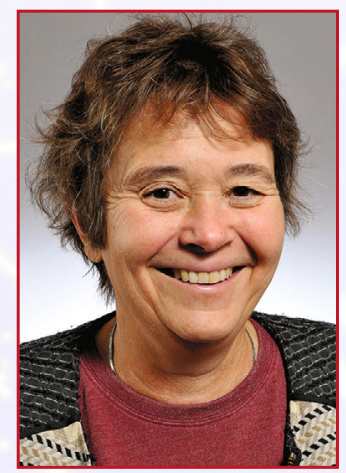

Dorte Juul Jensen

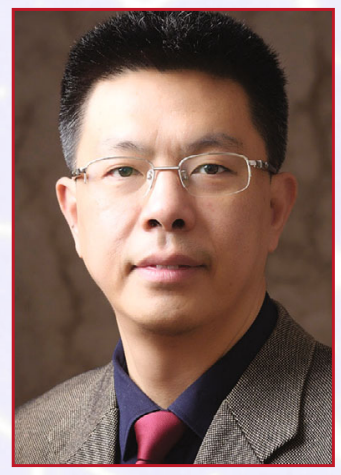

Lei Zhang

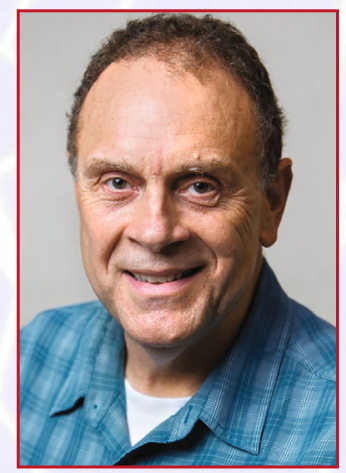

Dorte Juul Jensen, Technical University of Denmark: "For me, the 3DMS congress series is outstanding and a must to attend, as it is the cornerstone in building a new community within materials science. This community has grown significantly over the years, and it is enlightening that such a broad and cross-disciplinary 'team' has built up. Without the various skills, we would never have been able to move materials science to 3D."

\section{Lei Zhang, Chinese Academy of Sciences: "From my personal point of view, the 3DMS congresses give me a good opportunity to meet worldwide colleagues who have common interests in promoting the application of 3D techniques and methodology in materials science research."}

\section{Matthew P. Miller, Cornell University} and Cornell High Energy Synchrotron Source: "The most exciting thing for me about 3DMS is watching how many of the early 3D experiments-often conducted on model materials-have become reliable $3 \mathrm{D}$ material measurements, capable of quantifying the evolving microstructure of real materials in some of the most important and challenging real applications a material can face. In the eight years of 3DMS, the science has shifted from the characterization probe to the material itself."

\section{The Continuing Impact of 3DMS}

Looking forward, it will be important to focus our work on the full 3D information. It is an emerging trend that machine learning can boost simulations with abundant big data produced by the digitalized model of real materials with 3D overall configuration of phases, grains, internal defects, composite structures, etc. Only by using the full 3D information, a correct image can be obtained.

The applications of 3D are increasingly covering all aspects, from basic bluesky research to practical industrial applications. We consider it of importance to advance the ongoing research with a $3 \mathrm{D}$ focus but also to apply the methods even more broadly. We expect to see more 3D tools being developed for use in the home laboratories to supplement the more advanced experiments at large international facilities. Altogether, this will contribute to improving simulation tools and predictive capabilities in the advancement of existing materials and in the development of new materials and manufacturing routes, including additive manufacturing. Finally, it will be fascinating to discover the potential of the most advanced tools, such as incorporating machine learning and deep learning technologies.

Since the congress was established, 3DMS has been essential to addressing some critical problems in the 3D space. Advancements will continue through the fifth iteration and future iterations as the community grows and encourages technology advancements and research collaborations.

Dorte Juul Jensen is a professor at the Technical University of Denmark; Matthew Miller is an engineering professor at Cornell University and associate director of the Cornell High Energy Synchrotron Source; and Lei Zhang is a professor at the Shenyang National Laboratory for Materials Science, Chinese Academy of Sciences. Juul Jensen is the chair of the 3DMS 2020 organizing committee, with Miller and Zhang both also serving as congress organizers.

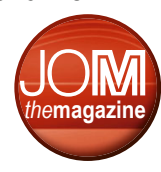

